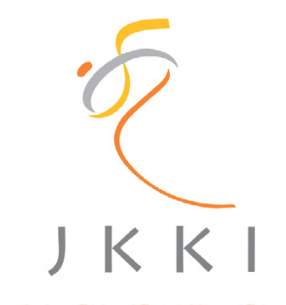

Jurnal Kedokteran dan Kesehatan Indonesia

Indonesian Journal of Medicine and Health

Journal homepage : www.journal.uii.ac.id/index.php/jkki

\title{
The comparison of transcatheter aortic valve implantation (TAVI) and surgical aortic valve replacement (SAVR)
}

Yuliana Yakobus*1

${ }^{1}$ Department of Cardiac Surgery, University Hospital Halle, Halle (Saale), Germany

\begin{tabular}{|c|c|}
\hline & \\
\hline 50 & \multirow{4}{*}{$\begin{array}{l}\text { Whe use of transcatheter aortic valve implantation (TAVI) to treat } \\
\text { severe symptomatic aortic valve stenosis has increased in the } \\
\text { last decade. In the past, medical therapy with or without balloon } \\
\text { aortic valvuloplasty was the only option for inoperable patients. More } \\
\text { recently, TAVI has become the treatment of choice for these patients and } \\
\text { the preferred alternative for high-risk operable patients. Available data } \\
\text { from randomised controlled trials and large registries in elderly patients } \\
\text { at increased surgical risk show that TAVI is superior regarding mortality } \\
\text { to medical therapy in extreme-risk patients, non-inferior or superior to } \\
\text { surgery in high-risk patients and noninferior to surgery and even superior } \\
\text { when transfemoral access is possible in intermediate-risk patients. }{ }^{1}\end{array}$} \\
\hline & \\
\hline 2 & \\
\hline & \\
\hline
\end{tabular}

\section{Indications}

Indications for aortic valve replacement (surgical or transcatheter) are as follows: ${ }^{2}$

1. Severe high-gradient aortic stenosis (AS) with symptoms (class I recommendation, level B evidence).

2. Asymptomatic patients with severe AS and left ventricular ejection fraction (LVEF) $<50$ (class I recommendation, level B evidence).

3. Severe AS when undergoing other cardiac surgery (class I recommendation, level B evidence).

4. Asymptomatic severe AS and low surgical risk (class IIa recommendation, level B evidence).

5. Symptomatic with low-flow/low-gradient severe AS (class IIa recommendation, level B evidence).

6. Moderate AS and undergoing other cardiac surgery (class IIa recommendation, level C evidence).

Aspects to be considered by the Heart Team for the decision between SAVR and TAVI in patients with severe aortic stenosis and increased surgical risk: ${ }^{3}$

Favours TAVI Favours SAVR

\section{Clinical characteristics}

The Society of Thoracic Surgeons/The European System for Cardiac

Operative Risk Evaluation II (STS/Euro-SCORE II) $<4 \%$

(Logistic Euro-SCORE I $<10 \%$ )

STS/Euro-SCORE II $\geq 4 \%$

(Logistic Euro-SCORE I $\geq 10 \%$ )

Presence of severe comorbidity

(not adequately reflected by scores)

Age $<75$ years

Age $\geq 75$ years 
Favours TAVI Favours SAVR

Previous cardiac surgery

Frailty

Restricted mobility and conditions that may affect the rehabilitation process after the procedure

Suspicion of endocarditis

\section{Anatomical and technical aspects}

Favourable access for transfemoral TAVI

Unfavourable access (any) for TAVI

Sequelae of chest radiation

Porcelain aorta

Presence of intact coronary bypass grafts at risk when sternotomy is performed

Expected patient-prosthesis mismatch

Severe chest deformation or scoliosis

Short distance between coronary ostia and aortic valve annulus

Size of aortic valve annulus out of range for TAVI

Aortic root unfavourable for TAVI

Valve morphology (bicuspid, degree of calcification, calcification pattern) unfavourable for TAVI

Presence of thrombi in Aorta or left ventricle
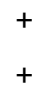

$+$

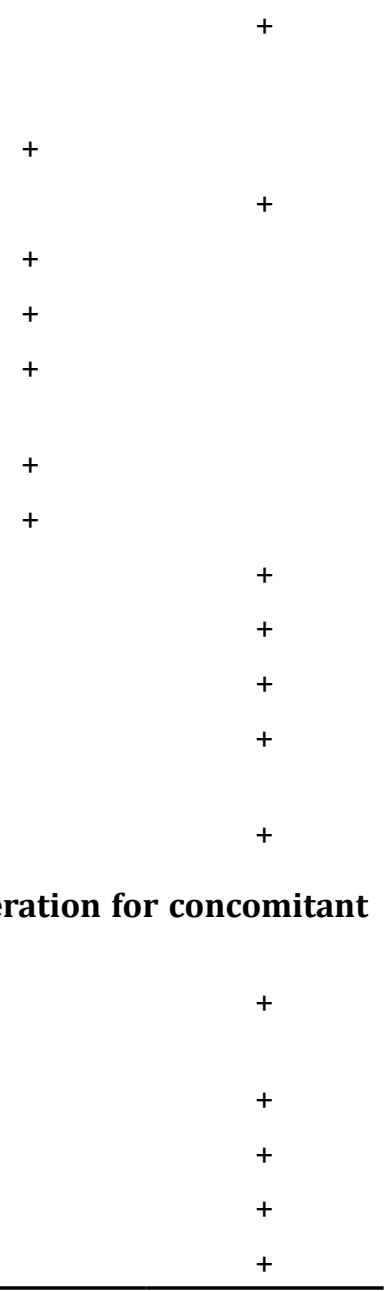

Cardiac conditions in additions to aortic stenosis that require consideration for concomitant
intervention
Severe coronary artery disease (CAD) requiring revascularization by
coronary artery bypass grafting (CABG)
Severe primary mitral valve disease, which could be treated surgically
Severe tricuspid valve disease

Contraindications

Contraindications for transcatheter aortic valve replacement are as follows: ${ }^{2}$

Life expectancy less than 12 months owing to a noncardiac cause, myocardial infarction within the last 30 days, congenital unicuspid, bicuspid or noncalcified valve, hypertrophic cardiomyopathy, need for emergency surgery, left ventricular ejection fraction less than $20 \%$, severe pulmonary hypertension with right ventricular dysfunction, echocardiographic evidence of intracardiac mass, thrombus or vegetation, native aortic annulus smaller than $18 \mathrm{~mm}$ or larger than $25 \mathrm{~mm}$, severe mitral regurgitation, magnetic resonance imaging (MRI) confirmed cerebrovascular accident (CVA) or transient ischemic attack (TIA) within last six months, end-stage renal disease, mixed aortic valve disease (concomitant aortic regurgitation), or significant aortic disease.

\section{The concept of the Heart Team}

The concept of Heart Team management of heart valve disease has been well-published and wellestablished. A typical valvular Heart Team consists of the cardiothoracic surgeon, the interventional cardiologist, the anesthetists, a structural heart disease expert, and imaging specialists, amongst others. 
A hybrid operating room is desired and recommended for TAVI procedures. ${ }^{4}$

The suitable therapy for the patient should be determined by the Heart Team. It is crucial that Heart Team takes the available scientific experience as well as recommendations from guidelines into account when deciding on an individual patient's therapy, they also need to reflect on center-specific outcomes of the various treatment options such as TAVI and SAVR. A treatment strategy that may work very well in one centermay not be appropriate in another center.

Heart Team needs to be able to work in an environment that provides comprehensive diagnostics and treatment options. "Heart Valve Centers", in which a multidisciplinary team works together on a regular basis and as a result has established appropriate communication structures, seem to be ideally placed to become centers of excellence for the treatment of valvular heart disease in the future. Regarding treatment, surgical and interventional procedures such as valve repair, SAVR, aortic root surgery and TAVI should be routine. For an adequate assessment before treatment, it is paramount that these centers provide a comprehensive diagnostic armament of the highest quality. This should include 3D, stress and perioperative transesophageal echocardiography, cardiac computed tomography (CT), cardiac MRI and positron emission tomography $\mathrm{CT}^{3}$

\section{Antithrombotic therapy}

The antithrombotic therapy post-TAVI prevents cerebral ischaemic events. Based on the experience of SAVR: with post-procedural sinus rhythm, dual antiplatelet therapy with clopidogrel and aspirin for 3-6 months followed by lifelong aspirin therapy is recommended. Clopidogrel should not be used if a vitamin $\mathrm{K}$ antagonist is used. ${ }^{5}$

\section{REFERENCES}

1. Falk V, Baumgartner H, Bax JJ, De Bonis M, Hamm C, Holm PJ, et al. 2017 ESC/EACTS guidelines for the management of valvular heart disease. European Journal of Cardio-Thoracic Surgery . 2017;52(4):616-64.

2. Mahmaljy H , Bhimji SS. Aortic valve, transcatheter replacement (TAVR/TAVI, percutaneous replacement). StatPearls Publishing LLC. 2018.

3. Khosravi A, Wendler O. TAVI 2018: From guidelines to practice. European Journal of Cardiology Practice. 2018;15(29).

4. Baumbach H, Wachter K, Rustenbach C, Ursulescu A, Franke UFW. Transcatheter aortic valve implantation. Annals of Cardiothoracic Surgery. 2017;6(5):455-62.

5. Hamm CW, Arsalan M, Mack MJ. The future of transcatheter aortic valve implantation. European Heart Journal. 2016;37(10):803-10. 